\title{
ARTICLE Cognitive deficit in methamphetamine users relative to childhood academic performance: link to cortical thickness
}

\author{
Andy C. Dean ${ }^{1,2}$, Angelica M. Morales ${ }^{1}$, Gerhard Hellemann $\mathbb{D}^{1}$ and Edythe D. London ${ }^{1,3,2}$
}

Individuals with cognitive problems may be predisposed to develop substance use disorders; therefore, differences in cognitive function between methamphetamine users and control participants may be attributable to premorbid factors rather than methamphetamine use. The goal of this study was to clarify the extent to which this is the case. Childhood academic transcripts were obtained for 37 methamphetamine-dependent adults and 41 control participants of similar educational level and premorbid IQ. Each participant completed a comprehensive cognitive battery and received a structural magnetic resonance imaging scan. Data from control participants and linear regression were used to develop a normative model to describe the relationship between childhood academic performance and scores on the cognitive battery. Using this model, cognitive performance of methamphetamine users was predicted from their premorbid academic scores. Results indicated that methamphetamine users' childhood grade point average was significantly lower than that of the control group $(p<0.05)$. Further, methamphetamine users overall cognitive performance was lower than was predicted from their grade point average prior to methamphetamine use $(p=$ 0.001 ), with specific deficits in attention/concentration and memory ( $p s<0.01)$. Memory deficits were associated with lower wholebrain cortical thickness $(p<0.05)$. Thus, in addition to having an apparent premorbid weakness in cognition, methamphetamine users exhibit subsequent cognitive function that is significantly lower than premorbid estimates would predict. The results support the view that chronic methamphetamine use causes a decline in cognition and/or a failure to develop normative cognitive abilities, although aside from methamphetamine use per se, other drug use and unidentified factors likely contribute to the observed effects.

Neuropsychopharmacology (2018) 43:1745-1752; https://doi.org/10.1038/s41386-018-0065-1

\section{INTRODUCTION}

Chronic use of methamphetamine has been associated with cognitive deficits. Meta-analytic reviews indicate that methamphetamine users have deficits relative to healthy control subjects in multiple cognitive functions, including the domains of learning, memory, executive function, processing speed and attention $[1,2]$. Effect sizes for these deficits generally have been in the medium range (e.g., ranging from $d=-0.34$ in language to $d=-0.66$ in learning; [2]).

Deficits in the cognitive performance of methamphetamine users have typically been interpreted as reflecting neurotoxic consequences of methamphetamine misuse; however, some evidence has called this conclusion into question. In longitudinal studies, some substance users have exhibited impairments in executive functioning as children, before they abused drugs, and these deficits may have conferred risk for later drug use $([3,4]$; see also [5]). Studies of siblings have shown that stimulant users have deficits in inhibitory control that are comparable to those of their brothers/sisters who do not use drugs, suggesting that the deficits are not attributable to drug use $[6,7]$. On the other hand, stimulant users perform significantly worse than their siblings who do not use drugs in the domain of executive functioning, consistent with a drug-induced effect [7]. Among twins who were discordant for weekly amphetamine use, the amphetamine users underperformed their twins on tests of processing speed and motor function, whereas the non-drug-using twins performed worse than the amphetamine users on a test of sustained attention [8]. These observations collectively suggest that at least some of the cognitive deficits observed in methamphetamine users reflect pre-existing deficiencies rather than substanceinduced decline.

Although it would be informative to evaluate the cognitive functioning of individuals before and after years of methamphetamine abuse to test for drug-induced decline, such study designs are costly and methodologically difficult, especially because the base rate of methamphetamine use is low relative to other drugs, such as alcohol and marijuana (but see the recently initiated Adolescent Brain Cognitive Development Study; https:// addictionresearch.nih.gov/abcd-study). It is also possible, however, to use historical data to estimate an individual's expected cognitive trajectory. Because childhood academic performance is highly correlated with later cognitive functioning [9-11], childhood academic performance can be used to predict a person's expected cognitive function later in life. High school cumulative grade point average (GPA), in particular, is correlated with most cognitive measures in a comprehensive neuropsychological battery administered in adulthood, with the strongest correlations $(r=0.50-0.40)$ involving measures of executive functioning,

\footnotetext{
${ }^{1}$ Department of Psychiatry and Biobehavioral Sciences, UCLA Semel Institute for Neuroscience, David Geffen School of Medicine, Los Angeles, CA 90024, USA; ${ }^{2}$ Brain Research

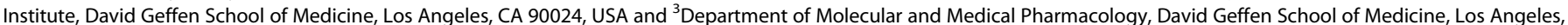
CA 90024, USA

Correspondence: Andy C. Dean (acdean@mednet.ucla.edu)
}

Received: 29 January 2018 Revised: 26 March 2018 Accepted: 4 April 2018

Published online: 20 April 2018 
immediate memory and processing speed, and modest to nonsignificant relationships with measures of delayed recall and motor function [12]. These associations are consistent with the broader literature showing strong relationships $(r=0.50-0.40)$ between measures of intelligence and GPA (for review, see [13]). Although predictive models using childhood academic scores have been helpful in evaluating the cognitive effects of psychiatric conditions, such as schizophrenia [14], these techniques have not been applied toward understanding the effects of methamphetamine abuse.

To provide an improved estimate of potentially methamphetamine-induced cognitive decline, childhood academic transcripts were obtained from methamphetamine-dependent participants $(N=37)$ so that their adult cognitive performance could be predicted from their academic scores, using a normative statistical model developed in healthy control subjects $(N=41)$. Based on the aforementioned literature, it was hypothesized that the methamphetamine users would have a lower childhood GPA than the healthy control subjects, but would also show a deficit in current cognitive performance relative to that predicted from their childhood GPA.

Given that the aforementioned procedure could improve upon previous estimates of methamphetamine-associated cognitive deficits (e.g., relative to raw cognitive test scores), we also examined the relationship between cognitive deficits and cerebral cortical thickness measured with magnetic resonance imaging (MRI). In adolescent methamphetamine users, executive functioning scores are negatively correlated with global cortical thickness [15]. In the current study of adults, deficits in specific cognitive domains (predicted minus actual scores) were tested for relationships with cortical thickness, with the hypothesis that deficits would be associated with less thickness in whole-brain and/or in regions of multi-modal association cortex (see [16]).

\section{METHODS AND MATERIALS}

\section{Participants}

The participants were 41 healthy control and 37 methamphetamine-dependent subjects who were not seeking treatment. Data on cognitive performance of these subjects have not been previously published; group differences in cortical thickness were previously examined from a subset of the subjects ( 70\%) [17]. The participants were recruited using Internet and local newspaper advertisements, and they received monetary compensation in return for maintaining abstinence from drugs and completing research procedures. After receiving a detailed description of the protocol, they provided written informed consent, following the guidelines of the UCLA Office for Protection of Research Subjects. Twenty-two methamphetamine users completed the study while residing on a research ward at the UCLA General Clinical Research Center (GCRC), and 15 completed the study as outpatients after closure of the GCRC (residential and outpatient methamphetamine users did not differ in overall cognitive battery scores, $p>$ $0.05)$. All control subjects were assessed on a nonresidential basis. The methamphetamine users tested positive for methamphetamine in urinalysis at study entry, but negative for methamphetamine and other drugs of abuse (amphetamine, opiates, cocaine, benzodiazepines) on days of cognitive and MRI assessment, following an average of 6.9 days of abstinence $(S D=2.7$; range $3-30)$. At all time points, the control subjects provided tests that were negative for drugs, except marijuana. Given the long duration in which cannabinoids can be detected in urine, brief abstinence from marijuana for nonresidential participants was verified through saliva testing (Oratect; Grapevine, Texas), with all participants endorsing at least 3 days of abstinence from drugs at the time of testing. Abstinence for residential subjects was supervised. All participants were fluent in English and were administered the Structured Clinical Interview for the DSM-IV for
Axis I diagnosis [18]. The exclusion criteria, based on interview and laboratory tests, were: neurological disease (e.g., stroke, head trauma with loss of consciousness $>30 \mathrm{~min}$ ); frank structural brain abnormalities on MRl; systemic, cardiovascular, or pulmonary disease; HIV infection (HIV1/HIV2 antibody screen); abnormal laboratory test findings (hematocrit, plasma electrolytes, markers for hepatic and renal function); use of psychotropic medications; diagnosis of current abuse or dependence for any substance other than methamphetamine, marijuana, or nicotine; and any current non-substance-induced Axis I psychiatric conditions (with the exception of one methamphetamine user with current social phobia). Six participants met criteria for current marijuana abuse or dependence, two in the control group and four in the methamphetamine group.

In terms of past (lifetime) psychiatric diagnoses, six methamphetamine users and seven control subjects met criteria for past major depression (in full remission at the time of this study); one methamphetamine user had a past hypomanic episode (former bipolar II) and one had past PTSD (in remission). Methamphetamine users were also more likely than control subjects to have met criteria for past substance abuse or dependence diagnoses (see Table 1). All participants who had past diagnoses were in sustained full remission from the respective disorders (at least 1 year without symptoms, but typically 4-10 years symptom-free, based on available data), except for three methamphetamine users who had past drug-use diagnoses in early full remission (at least 1 month abstinent but $<1$ year; two with former alcohol abuse, and one with former cannabis dependence).

\section{Measures/procedures}

Academic records. Participants provided the names and, if possible, locations of all schools they had attended. With consent, schools were contacted to request all grade transcripts and standardized test scores available between the elementary and high school years, excluding college. A mean GPA was calculated for each subject, by taking the average of all the course grades that were available. For uniformity across schools, course grades were coded $4(A+$ to $A-), 3(B+$ to $B-), 2(C+$ to $C-), 1$ (D + to $D-)$, and $0(F)$, consistent with the coding scheme of the U.S. Department of Education [19]. For methamphetamine users, the average of course grading was taken for years prior to the date of their first reported methamphetamine use. Across subjects, grade information was available for a range of between 1 and 13 years (note that some participants repeated grades). Even given the premethamphetamine use adjustment, the groups did not significantly differ in the number of years of data used for calculations (methamphetamine mean $4.3, \mathrm{SD}=2.0$; control mean $5.2, \mathrm{SD}=$ $2.7 ; p>.10$ ). For a smaller subset of participants (control $N=19$; methamphetamine $N=24$ ), at least one or more standardized test scores were available (prior to methamphetamine use); these included tests such as the Scholastic Aptitude Test, lowa Test of Basic Skills, the American College Testing exam, and the California Test of Basic Skills. The mean of the normative percentiles for available standardized tests was calculated for each subject (see Table 1). GPA and mean standardized test performance were positively correlated $(r=0.45, p=0.002)$.

Cognitive battery. Cognitive assessment was conducted by clinical psychology doctoral students trained by the first author (A.C.D.). In order to avoid fatigue, testing was performed on 2 separate days and included breaks as needed. During the assessment, all participants performed satisfactorily on a standalone measure of effort (Victoria Symptom Validity Test; [20]), as well as embedded measures of effort (e.g., digit span age corrected score; trailmaking part A cutoffs; [21, 22]), guarding against the possibility that low scores could reflect insufficient effort on the part of the examinee. The cognitive battery included measures of attention/concentration: Trailmaking Part A [23], 
Table 1. Characteristics of research participants

\begin{tabular}{|c|c|c|}
\hline & $\begin{array}{l}\text { Healthy } \\
\text { control }\end{array}$ & $\begin{array}{l}\text { Methamphetamine- } \\
\text { dependent }\end{array}$ \\
\hline Sample size & 41 & 37 \\
\hline Age & $33.7 \pm 7.6$ & $34.0 \pm 8.9$ \\
\hline Education (years) & $12.7 \pm 1.4$ & $12.6 \pm 1.7$ \\
\hline Estimated full scale IQ & $\begin{array}{l}105.0 \pm \\
9.7\end{array}$ & $104.9 \pm 8.4$ \\
\hline Childhood economic class (self-rated) & $2.5 \pm 1.0$ & $2.4 \pm 0.9$ \\
\hline Highest parental education (years) & $13.9 \pm 2.6$ & $12.9 \pm 3.2$ \\
\hline Combined parental income & $8.6 \pm 5.3$ & $7.7 \pm 4.0$ \\
\hline $\begin{array}{l}\text { Average of median household income } \\
\text { of participants' school locations }\end{array}$ & $\begin{array}{l}\$ 57,929 \\
\pm 19,549\end{array}$ & $\$ 62,101 \pm 21,445$ \\
\hline \multicolumn{3}{|c|}{ Ethnicity } \\
\hline Caucasian & 13 & 12 \\
\hline African Am & 8 & 3 \\
\hline Hispanic & 15 & 14 \\
\hline Asian/Pacific Islander & 3 & 3 \\
\hline \multicolumn{3}{|l|}{ Other } \\
\hline \multicolumn{3}{|l|}{ Gender } \\
\hline Male/female & $21 / 20$ & $25 / 12$ \\
\hline Employed (yes/no) & $24 / 17$ & $10 / 27^{* *}$ \\
\hline Cigarette smokers (yes/no) & $14 / 27$ & $34 / 3 * *$ \\
\hline Cigarette pack-years (smokers only) & $10.3 \pm 7.8$ & $9.8 \pm 9.9$ \\
\hline Days alcohol/past 30 & $2.3 \pm 4.1$ & $3.4 \pm 4.5$ \\
\hline Days marijuana/past 30 & $2.5 \pm 6.8$ & $3.9 \pm 8.5$ \\
\hline $\begin{array}{l}\text { Lifetime alcohol abuse or } \\
\text { dependence }\end{array}$ & 9 & $26^{* *}$ \\
\hline $\begin{array}{l}\text { Lifetime cocaine abuse or } \\
\text { dependence }\end{array}$ & 0 & $12 * *$ \\
\hline $\begin{array}{l}\text { Lifetime marijuana abuse or } \\
\text { dependence }\end{array}$ & 9 & $21^{* *}$ \\
\hline $\begin{array}{l}\text { Lifetime other drug abuse or } \\
\text { dependence }\end{array}$ & 0 & $9 * *$ \\
\hline Days methamphetamine/past 30 & -- & $21.9 \pm 8.2$ \\
\hline $\begin{array}{l}\text { Duration of heavy } \\
\text { methamphetamine use (year) }\end{array}$ & -- & $6.5 \pm 6.0$ \\
\hline Grams methamphetamine/day & -- & $1.1 \pm 0.8$ \\
\hline Childhood grade point average & $2.5 \pm 0.6$ & $2.1 \pm 0.7^{*}$ \\
\hline $\begin{array}{l}\text { Average childhood standardized test } \\
\text { score (percentile) }\end{array}$ & $\begin{array}{l}58.5 \pm \\
23.7\end{array}$ & $48.7 \pm 22.7$ \\
\hline
\end{tabular}

Values are means $\pm S D s$, where appropriate. The symbols * and ** indicate significant difference from the control group at $p<0.05$ and $p<0.01$, respectively. IQ was estimated with the Wechsler Test of Adult Reading. For self-rated economic class, $1=$ lower class, $2=$ lower middle class, $3=$ middle class, $4=$ upper middle class, $5=$ upper class. For combined parental income, $7=\$ 60,000-70,000$ per year, $8=70,000-80,000$ per year (in U.S. dollars). Heavy methamphetamine use defined as using at least three times a week, or twice weekly binges. Childhood Grade Point Average and standardized test scores for methamphetamine users were calculated prior to the onset of their methamphetamine use. Childhood standardized test scores were only available from a subset of participants (control $N=19$; methamphetamine $N=24$ ).

Stroop Color and Word identification [24], Digit Symbol Coding [25], Digit Span [25], Continuous Performance Test [26, 27], Wisconsin Card Sorting-Failure to Maintain Set [28], Brief Test of Attention [29], and Spatial Capacity Delayed Response Test [27, 30]; learning/memory: Selective Reminding Test [31], Rey Auditory Verbal Learning Test [32], and the Brief Visuospatial Memory
Test-Revised [33]; and executive function: Stroop Color-Word Interference [24], Trailmaking Part B [23], Controlled Oral Word Fluency and Category Switching [34], Wisconsin Card SortingPerseverative Errors [28], Attention Networks Task [27, 35], and the Stop Signal Task [36]. As described previously [37-39], an overall cognitive battery score was created by centering and scaling each of the test scores based on the mean and standard deviation of the control group, and then averaging the resulting standardized scores (tests on which lower scores indicated better performance were multiplied by -1 to keep the directionality of the measures consistent). Domain-specific scores (i.e., attention/concentration, learning/memory, executive function) were similarly generated. Standardizing to the control sample is simply a scale change applied equally to all subjects, designed to put equal weights on the measures in the composite score. It does not represent selfreferential use of the data or influence predictive modeling. Standardizing to the control group, rather than published norms, is preferable so that variations in performance are not introduced by differences in normative datasets used for different tests (see [40]).

Self-report measures. On each day of cognitive assessment, methamphetamine withdrawal was assessed with the 16-item, Likert-scale Amphetamine Cessation Symptom Assessment [41]. Because the two total scores were highly correlated $(r=0.84, p<$ $0.0001)$, they were averaged when tested for association with cognitive deficit scores (see Results).

Other demographic and drug-use information was obtained with standard laboratory forms at intake. Amount of methamphetamine use was queried as the "average amount" the individual used on days that he/she used methamphetamine (in grams).

\section{Structural MRI}

After excluding subjects with MRI contraindications (e.g., claustrophobia or metallic implants) and motion artifact (visual distortion of the structural image), 31 methamphetamine users and 31 controls had useable MRI data. High-resolution magnetization-prepared rapid gradient imaging was performed at 1.5 Tesla on a Siemens Sonata scanner ( $T R=1900 \mathrm{~ms}$, $T E=4.38 \mathrm{~ms}$, flip angle $=15^{\circ}$, voxel size $=1 \mathrm{~mm}^{3}$ ). Cortical thickness was measured using the FreeSurfer software package (version 5.3) and images were bias-field corrected and non-brain tissue removed [42]. To generate cortical surfaces, a tessellation was formed along the white-matter surface and was grown outward toward the intensity gradient separating the gray matter from the cerebrospinal fluid. White-matter and pial surfaces were visually inspected for accuracy, and manually corrected in cases where the white matter was not accurately classified and the pial surface included dura, sinus, or skull.

\section{Data analysis}

Demographic differences between groups were evaluated using $t$ tests or $x^{2}$-tests, as appropriate. To create a normative model of the relationship between academic scores and overall cognition, bivariate relationships (i.e., correlations, $t$-tests and analysis of variance, as appropriate) were first explored between overall cognitive battery scores, demographics, and childhood GPA in control subjects. Demographic variables considered here were those that would not be affected by potential methamphetamine use in an individual (e.g., age, gender, ethnicity, parental factors), unlike years of education (e.g., high school dropout is prospectively predicted by methamphetamine use [43]). Once significant variables were identified in bivariate relationships, the overall cognitive battery was linearly regressed onto these variables in control subjects. Then, similar to other predictive modeling [39], using the resulting regression equation of the form $Y=$ unstandardized constant + variable $1^{*}\left(\beta_{1}\right)+$ variable $2^{*}\left(\beta_{2}\right)$, etc., a 
predicted cognitive battery score was calculated for each methamphetamine user by plugging his/her values into the equation. The difference between predicted versus actual cognitive battery scores in methamphetamine users was evaluated with a paired $t$-test. Overall cognitive deficit scores for each methamphetamine user were calculated by subtracting predicted cognitive battery scores from actual cognitive battery scores. Deficit scores in each cognitive domain (i.e., attention/concentration, learning/memory, executive function) were similarly generated by creating separate regression equations with cognitive domain scores as the dependent variable, using the covariates implemented in the overall analysis.

To evaluate group differences in cortical thickness, an omnibus test was first run using the General Linear Mixed Model (GLMM), with thickness as the dependent variable, group as a betweensubjects variable, and hemisphere ( 2 ; right or left) as a withinsubject variable. If this model was significant, post hoc GLMMs were run to determine which hemisphere (and subsequently brain regions, if appropriate) drove omnibus findings.

In the methamphetamine group alone, the relationship between deficits in specific cognitive domains (domains in which performance was lower than predicted) and cortical thickness was analyzed with GLMM (with separate models for each respective domain). Models began with an omnibus test using thickness as the dependent variable, domain deficit as a between-subjects factor, and hemisphere (2; right or left) as a within-subject factor. If domain deficit or deficit by hemisphere interactions were present in the omnibus model, post hocGLMMs were conducted to determine which hemisphere (and subsequently brain regions, if appropriate) drove omnibus findings.

\section{RESULTS}

Group comparisons of demographic characteristics

The methamphetamine users and the healthy control participants did not significantly differ in age, gender, years of education, estimated IQ (Wechsler Test of Adult Reading, [44]) (also indicated to function as a proxy for quality of education, see $[45,46])$, ethnicity, self-rated childhood economic class, highest parental education, combined parental income, average median household income of the location (zip codes) in which schools were attended, days of alcohol use in the last 30 days, or days of marijuana use in the last 30 days ( $p s>0.05$ ). Members of the methamphetamine group were more likely to be unemployed, and the group included a higher proportion of cigarette smokers than the control group ( $p s<0.01$ ), although smokers in the two groups did not differ in cumulative smoking (pack-years, $p>0.05$ ). Methamphetamine users were more likely to have past diagnoses (in full remission) of alcohol, cocaine, marijuana, and "other" drug abuse or dependence than control subjects ( $p s<0.01$ ); however, in the methamphetamine group, the presence of these past diagnoses were not independently associated with cognitive battery scores (ps $>0.35$ ). Further, methamphetamine users without any past diagnoses $(N=6$; Cog battery mean $=-0.84$; $S D=0.77)$ exhibited nonsignificantly worse cognitive battery scores than those with past diagnoses $(N=31$; Cog battery mean $=-0.60 ; \mathrm{SD}=0.48, p$ $=0.30$ ).

The methamphetamine users had a lower childhood GPA (prior to using methamphetamine) than the control subjects ( $p=0.016$, see Table 1) and significantly lower cognitive battery scores $(p<$ 0.001). The two groups did not differ in average childhood standardized test scores $(p=0.178$, See Table 1$)$, although the smaller sample sizes available for this analysis limited statistical power.

Predictive modeling of cognition

In the data from control subjects, premorbid variables were explored for relationships with the overall cognitive battery score.
Gender, ethnicity, employment status, cigarette smoking status, childhood economic class, average household income from school locations, and highest parental education were not significantly related to overall cognition ( $p s>0.05$ ). In contrast, childhood GPA $(r=0.58, p<0.001)$ and combined parental income $(r=0.31, p=$ 0.048 ) were positively correlated with overall cognition, whereas age exhibited a trend-level negative relationship $(r=-0.30, p=$ 0.06). The relationships between overall cognition and GPA, parental income and age were best characterized as linear; quadratic terms did not improve model fit $(p s>0.05$ ). (Further, despite group differences in GPA, the ranges were overlapping (Control range $=0.875-3.729$; Methamphetamine range $=$ 0.911-3.414), indicating that predictive modeling would not need to extrapolate beyond available data].

Based on these findings, in the control subjects, the overall cognitive battery score was linearly regressed onto age, parental income, childhood GPA and cigarette smoking status (smoking status was included because the relationship with cognition was negative (nonsignificant) and most methamphetamine users smoke). The overall model was significant $\left(R^{2}=0.41, p<0.001\right)$. Using the unstandardized regression coefficients from this analysis $\left(Y=-0.886+\right.$ age $^{*}(-0.008)+$ parental income ${ }^{*}(0.025)+$ smoking status* $\left.(-0.200)+\mathrm{GPA}^{*}(0.404)\right)$, predicted cognitive battery scores were generated for the methamphetamine users. As shown in Fig. 1, these predicted battery scores were significantly higher (less negative) than the actual battery scores $(d=0.61, \mathrm{t}(36)=$ 3.732, $p=0.001$ ).

In the control subjects, regression models for each cognitive domain were significant (attention/concentration, $R^{2}=0.41, p=$ 0.001; memory/learning, $R^{2}=0.30, p=0.01$; executive function, $R^{2}$ $=0.38, p=0.001$ ), allowing for the prediction of domain scores. In methamphetamine users, predicted scores from these regressions were significantly higher (less negative) than their actual scores in the domain of attention/concentration $(d=0.69, \mathrm{t}(36)=4.177, p$ $<0.001)$ and memory $(d=0.46, \mathrm{t}(36)=2.805, p<0.01)$, but not

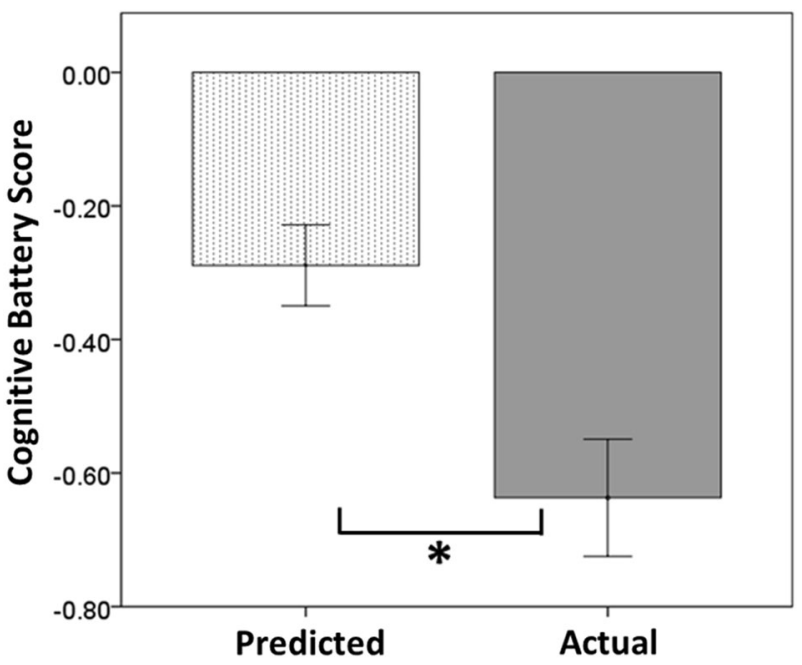

Fig. 1 Predicted versus actual cognitive battery scores for methamphetamine-dependent participants. Note: methamphetaminedependent participants $N=37$; Predicted cognitive battery scores were estimated from age, parental income, smoking status, and childhood grade point average (GPA) in school prior to methamphetamine use, using a regression equation originally defined in healthy control participants $(N=41)$. Scores on the $y$ axis reflect standard deviation units from the control sample's performance, with 0.0 indicating the mean score for control participants. Although predicted cognitive scores of methamphetamine users were below the control mean, their actual scores were significantly lower than this prediction (* ${ }^{*}$ paired $t$-test, $p=.001$ ). Error bars \pm 1 standard error 
executive functioning $(d=-0.27, \mathrm{t}(36)=-0.169, p=0.87)$, as shown in Fig. 2.

Methamphetamine-use and withdrawal variables were tested for relationships with overall and domain deficit scores (actual score minus predicted score). Methamphetamine withdrawal was negatively associated with the overall battery deficit score $(r=$ $-0.360, p=0.029)$; thus, greater withdrawal symptoms were associated with a larger cognitive deficit. However, when the variance associated with withdrawal was partialed out of cognitive battery scores, the difference between predicted and actual

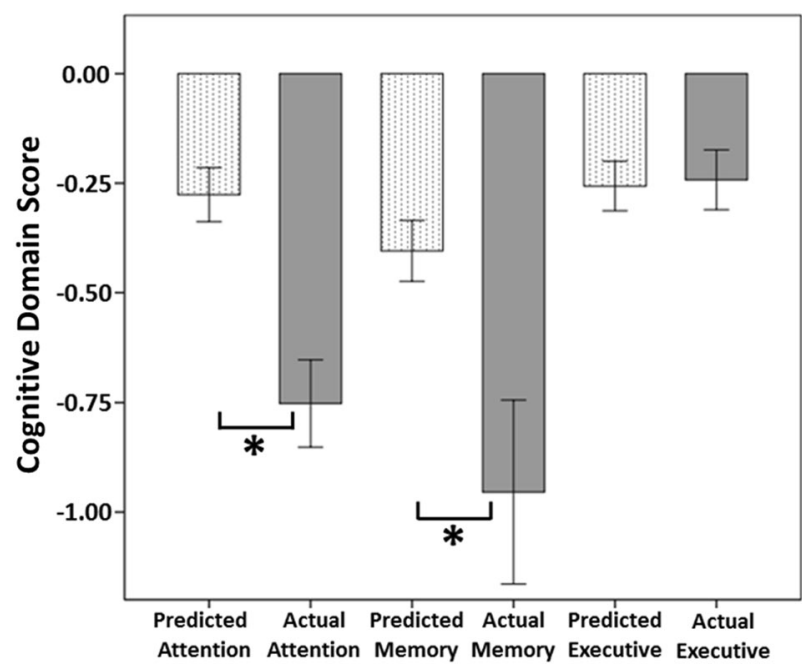

Fig. 2 Predicted versus actual cognitive domain scores for methamphetamine-dependent participants. Note: methamphetaminedependent participants $N=37$; Predicted cognitive domain scores (attention, memory, executive functioning) were estimated from demographics and childhood grade point average (GPA) in school prior to methamphetamine use, using regression equations originally defined in healthy control participants $(N=41$; see text). Scores on the $y$ axis reflect standard deviation units from the control sample's performance, with 0.0 indicating the mean score for control participants. Scores for methamphetamine users in the domains of attention and memory were significantly lower than predicted (*paired $t$-tests, $p s<.01$ ), whereas the domain of executive functioning was not lower than predicted $(p>.05)$. Error bars \pm 1 standard error overall cognitive battery scores remained statistically significant $(d$ $=0.54, \mathrm{t}(36)=3.302, p=0.002)$. Withdrawal scores were not significantly associated with attention and memory deficit scores (ps $>0.10)$.

Overall cognitive battery and attentional domain deficit scores were negatively related to the average amount of methamphetamine used per day (grams, $r=-0.397, p=0.045 ; r=-0.494, p$ $=0.010$; respectively), indicating that greater usage was associated with larger deficits. Cognitive deficit scores were unrelated to other methamphetamine-use variables, such as years and frequency of use ( $p s>0.05)$.

In the subset of data from participants who had standardized academic test scores available, a regression equation was created in the control subjects as before, except replacing GPA with average standardized test scores $\left(R^{2}=0.46, p=0.017\right)$. As before, using this method, the predicted cognitive battery scores of the methamphetamine users were significantly higher than their actual scores $(d=1.41, \mathrm{t}(23)=6.888, p<0.001)$, indicating that the effect was not particular to the use of GPA as an estimate of academic performance.

Finally, given that methamphetamine users and control subjects differed in childhood GPA, a subgroup analysis was conducted in which participants from each group were matched on GPA using the SPSS case-control matching algorithm (tolerance $=0.1$ ), resulting in 28 subjects from each group with similar GPAs (GPA mean difference $p=0.58$ ). Using regression in this subgroup, predicted cognitive battery scores for the methamphetamine users continued to be higher than their actual cognitive battery scores $(d=0.71, \mathrm{t}(36)=3.776, p=0.001)$.

\section{Cortical thickness}

In group comparisons of cortical thickness, there were no significant main effects of group or hemisphere, nor a significant interaction between group and hemisphere $(p s>0.05)$. The methamphetamine group exhibited marginally smaller thickness than control subjects ( $1 \%$ difference in the right hemisphere and $0.55 \%$ difference in the left), but these effects were not significant.

In the methamphetamine group alone, given observed deficits in the domains of attention/concentration and memory relative to premorbid predictions, the relationships between domain deficit scores (actual minus predicted) and cortical thickness were tested. In the attention/concentration analysis, there were no significant main effects of attention deficit score or hemisphere, nor a significant interaction between the two on thickness ( $p s>0.05)$. In
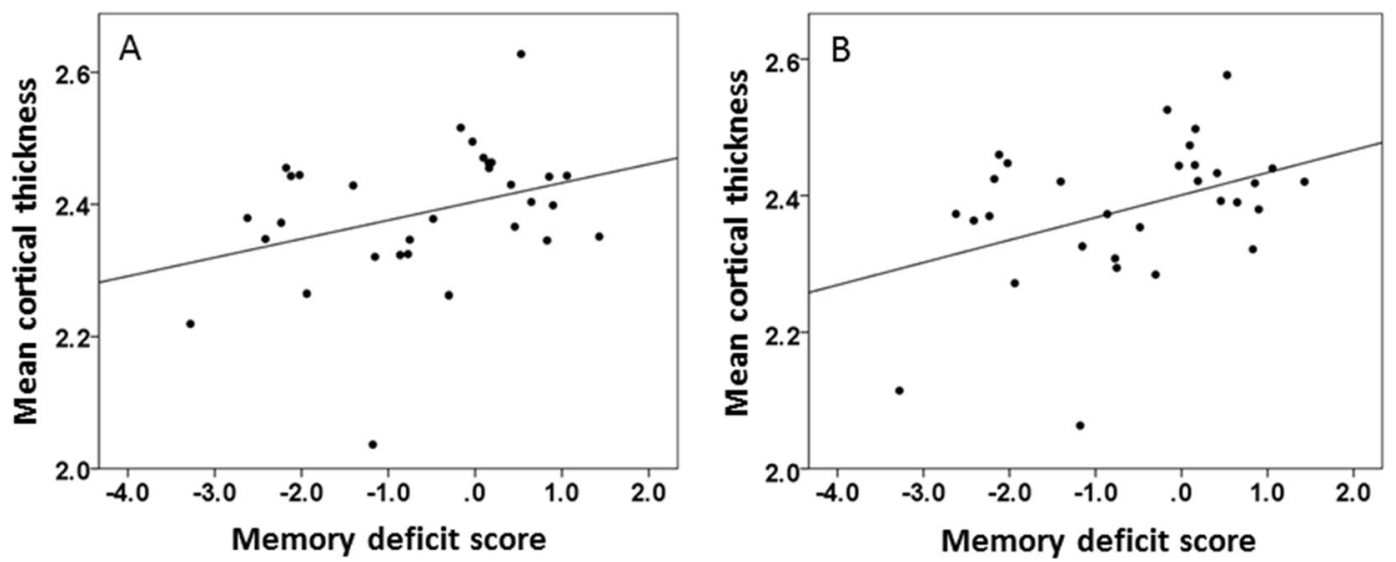

Fig. 3 Association between memory deficit scores and cortical thickness in methamphetamine users. Note: methamphetamine-dependent participants $N=31$. In omnibus analysis, whole-brain cortical thickness was significantly associated with memory deficit scores ( $p=0.037$, see text). a shows the relationship between memory deficit scores and mean cortical thickness of the left hemisphere $(r=0.34, p=0.060)$, whereas $\mathbf{b}$ shows the relationship between memory deficit scores and mean cortical thickness of the right hemisphere $(r=0.40, p=0.026)$. Larger deficits in memory (larger differences between actual and predicted memory scores, see text) were associated with smaller cortical thickness 
the memory deficit analysis, there was a significant main effect of memory deficit on thickness $(F(1,29)=4.771, p=0.037)$, without a significant effect of hemisphere or interaction between hemisphere and memory deficit ( $p s>0.05)$. Pearson correlation revealed that larger memory deficits were associated with lower cortical thickness in both hemispheres, although the effect was nonsignificantly stronger in the right hemisphere (see Fig. 3). In a follow-up GLMM in which cortical region was added as a withinsubject factor to the aforementioned GLMM (using 12 matched cortical regions per hemisphere, selected based on previous literature, see [47-52]), there was not a significant region by memory deficit interaction, nor a region by hemisphere by memory deficit interaction ( $p s>0.05$ ). This observation suggests that the relationship between memory deficit and cortical thickness was a global effect, not significantly driven by one or more particular cortical regions. However, for exploratory purposes, uncorrected correlations between memory deficit scores and specific cortical regions are presented in Supplementary Materials (Supplementary Table 1).

\section{DISCUSSION}

Examination of childhood academic transcripts revealed that methamphetamine users had significantly lower academic performance in school (GPA), prior to the onset of their methamphetamine use, than healthy comparison participants of similar educational level, estimated IQ and demographic characteristics. This finding supports prior evidence that substance users have premorbid cognitive limitations that predispose them to drug use $[3,4]$. Nonetheless, when predictive modeling was used to estimate expected cognitive functioning based on academic performance before methamphetamine use (as well as age, parental income level and cigarette smoking status), the methamphetamine users showed deficits in cognitive performance relative to the estimates. Thus, the methamphetamine users were performing at a lower cognitive level than would be expected from their premorbid function.

The reason that methamphetamine users underperform their predicted cognitive function is unknown. Findings from this study support the hypothesis that chronic methamphetamine use causes a decline in cognitive function, and/or a failure to develop normative cognitive abilities. Confidence in this conclusion is strengthened because the methamphetamine group and control group used for predictive modeling were generally well-matched on demographic characteristics (e.g., education, estimated IQ, ethnicity, several markers of socioeconomic status, see Table 1). Also supportive is the finding that larger overall cognitive deficit scores (actual scores minus predicted scores) were associated with higher self-reported average amounts of daily methamphetamine use. Animals administered methamphetamine using regimens that simulate human consumption of the drug also develop cognitive deficits (for review, see [53]).

Etiological factors other than methamphetamine use per se should be considered in explaining observed differences between predicted and actual cognitive scores. Overall deficit scores were negatively correlated with a measure of withdrawal on the days of testing, suggesting that methamphetamine withdrawal symptoms negatively affect cognitive functioning in early abstinence. However, when the effect of withdrawal on cognitive battery scores was statistically removed, a significant difference between predicted and actual cognitive battery scores remained (of moderate effect size, $d=0.54$ ), indicating that withdrawal symptoms do not fully account for predicted-observed differences.

Most methamphetamine users in the current study met criteria for only methamphetamine dependence (only a few had current comorbid marijuana abuse/dependence), but the majority also exhibited lifetime (past, in full remission) diagnoses of other drug abuse and dependence, including former use of alcohol, cocaine and marijuana. Whereas these past diagnoses were not independently associated with cognitive battery scores ( $p s>0.35$ ), it is difficult to disentangle effects of methamphetamine use from that of other substance use. This confound complicates the interpretation of differences between observed versus predicted cognitive scores, and presents a challenge to the field moving forward, even when robust longitudinal methods can be implemented to track changes in cognition over time (significant care will need to be taken to document drug use over time). To the extent that other drug use occurred prior to the onset of methamphetamine use, the results presented here may underestimate the effect of methamphetamine use on cognition because drug use may have negatively affected premorbid academic performance. If, on the other hand, other drug use co-occurred with methamphetamine use (in the past), the results may overestimate effects of methamphetamine use on cognition. Sufficient detail is not present in the data regarding past diagnoses to disentangle these different possibilities.

Consistent with a previous examination of a subsample of participants in the current study (of which $70 \%$ of participants overlap, [17]), cortical thickness did not differ between methamphetamine users and healthy control subjects. Memory deficit scores, however, were related to overall cortical thickness in methamphetamine users, less thickness being associated with more pronounced memory deficits. Meta-analytic data suggest that learning/memory may be particularly susceptible to methamphetamine (and possibly other drug) use [2], consistent with the findings here. The current data suggest that subtle alterations in large-scale cortical thickness may contribute to these memory problems. It is unclear, however, whether changes in cortical thickness have been induced by methamphetamine and other drug use or whether thickness reflects pre-existing phenomena. The latter possibility should be considered given that adolescent methamphetamine users have larger deficits in cortical thickness in several regions than adult methamphetamine users [15]. Smaller cortical thickness may thus function as a risk factor for the development of subsequent memory problems or other clinically relevant phenomena that could hamper treatment responsiveness. Notably, smaller cortical thickness in methamphetamine users has also been associated with stronger craving for the drug [17].

In comparison with premorbid estimates, attention/concentration and memory scores were significantly lower than predicted, whereas executive functioning scores were not. Relative to premorbid predictions, deficits in attention and memory had effect sizes in the medium range $(d=0.69$ and $d=0.46$, respectively; [54]; both effects were unassociated with withdrawal), consistent with meta-analytic data [2]. The finding that executive functioning scores in the methamphetamine users were not lower than predicted from premorbid variables was unexpected. Although this may suggest that executive deficits are premorbid in nature $[3,4]$, stimulant users (predominantly cocaine users but some amphetamine users) have exhibited worse performance on measures of executive functioning than their siblings who do not use drugs [7], suggesting a drug-induced effect. Variation in findings may be attributable to differences in the participants assessed, cognitive batteries used (e.g., Ersche used a fully computerized battery) and/or other differences in methodology. Additional research is needed to determine whether executive deficits found in methamphetamine users exceed those expected from premorbid findings.

This research is not without limitations. The methods used did not allow demonstration of a causal relationship between methamphetamine use and cognitive decline. Because GPA and the other predictors explained $\sim 40 \%$ of the variance in the overall cognitive scores in control subjects, the method used is an imperfect estimate that does not capture potentially important 
influences on cognitive function and development. At last, although sample sizes were reasonable in the context of challenges present to obtain the data (e.g., find and contact multiple schools, recruit groups with similar demographics, maintain abstinence in methamphetamine users and exclude current comorbid conditions), the size of the control sample was modest for the purpose of predicting cognitive scores. Replication is thus recommended to confirm results. Despite limitations, this study indicates that methamphetamine users have deficits in cognitive function relative to estimates of their premethamphetamine use level of function, and is consistent with the presence of both pre-existing and drug-induced cognitive effects.

\section{ACKNOWLEDGEMENTS}

We gratefully acknowledge the efforts of several graduate students and researchers who worked with study participants or research data, with whom this work would not have been possible: Ahoo Karimian, Psy.D; Alexandria Davis, MPA; Brittany Reid, Psy. D; Dara Ghahremani, Ph.D.; Edmon Artinyan, M.A.; Eric Wagreich; M.D.; Eugene Oh; Hannah Jones, Ph.D; Kimberly Lin; Kimberly Price; Kristina Mouzakis; Lori Meono, Psy. D; Maritza Johnson; Meredith Blackburn, M.A.; Milky Kohno, PhD; Sarah Klein, M.A.; and Thomas Hanson, Psy.D. We also thank Robert Bilder, Ph.D and Russel Poldrack, $\mathrm{Ph} . \mathrm{D}$ for their helpful comments on the associated grant application.

\section{FUNDING}

This research was supported by NIH grants K23 DA027734 (A.C.D.), R21 DA034928 (A C.D.), P20 DA022539 (E.D.L.), R01 DA020726 (E.D.L.), M01 RR00865 (UCLA GCRC), UL1TR000124 (UCLA CTSI), and endowments from the Thomas P and Katherine K Pike Chair in Addiction Studies and the Marjorie M Greene Trust (E.D.L.)

\section{ADDITIONAL INFORMATION}

The online version of this article (https://doi.org/10.1038/s41386-018-0065-1) contains supplementary material, which is available to authorized users.

Competing interest: The authors declare that they have no conflict of interest

Publisher's note: Springer Nature remains neutral with regard to jurisdictional claims in published maps and institutional affiliations.

\section{REFERENCES}

1. Fernandez-Serrano MJ, Perez-Garcia M, Verdejo-Garcia A. What are the specific vs. generalized effects of drugs of abuse on neuropsychological performance? Neurosci Biobehav Rev. 2011;35:377-406.

2. Scott JC, Woods SP, Matt GE, Meyer RA, Heaton RK, Atkinson JH et al. Neurocognitive effects of methamphetamine: a critical review and meta-analysis. Neuropsychol Rev. 2007;17:275-97.

3. Tarter RE, Kirisci L, Habeych M, Reynolds M, Vanyukov M. Neurobehavior disinhibition in childhood predisposes boys to substance use disorder by young adulthood: direct and mediated etiologic pathways. Drug Alcohol Depend. 2004;73:121-32.

4. Tarter RE, Kirisci L, Mezzich A, Cornelius JR, Pajer K, Vanyukov M et al. Neurobehavioral disinhibition in childhood predicts early age at onset of substance use disorder. Am J Psychiatry. 2003;160:1078-85.

5. Koenen KC, Moffitt TE, Roberts AL, Martin LT, Kubzansky L, Harrington $\mathrm{H}$ et al. Childhood IQ and adult mental disorders: a test of the cognitive reserve hypothesis. Am J Psychiatry. 2009;166:50-7.

6. Ersche KD, Jones PS, Williams GB, Turton AJ, Robbins TW, Bullmore ET. Abnormal brain structure implicated in stimulant drug addiction. Science. 2012a;335:601-4.

7. Ersche KD, Turton AJ, Chamberlain SR, Muller U, Bullmore ET, Robbins TW. Cognitive dysfunction and anxious-impulsive personality traits are endophenotypes for drug dependence. Am J Psychiatry. 2012b;169:926-36.

8. Toomey R, Lyons MJ, Eisen SA, Xian H, Chantarujikapong S, Seidman L et al. A twin study of the neuropsychological consequences of stimulant abuse. Arch Gen Psychiatry. 2003;60:303-10.

9. Baade LE, Schoenberg MR. A proposed method to estimate premorbid intelligence utilizing group achievement measures from school records. Arch Clin Neuropsychol. 2004;19:227-43.

10. Deary I, Strand S, Smith P, Fernades $C$. Intelligence and educational achievement. Intelligence. 2007;35:13-21.

11. Heaton RK, Pendleton MG. Use of Neuropsychological tests to predict adult patients' everyday functioning. J Consult Clin Psychol. 1981;49:807-21.
12. Greiffenstein MF, Baker WJ. Premorbid clues? Preinjury scholastic performance and present neuropsychological functioning in late postconcussion syndrome. Clin Neuropsychol. 2003;17:561-73.

13. Sternberg RJ, Grigorenko EL, Bundy DA. The predictive value of IQ. Merrill Palmer Q. 2001;47:1-41.

14. Bilder RM, Reiter G, Bates J, Lencz T, Szeszko P, Goldman RS et al. Cognitive development in schizophrenia: follow-back from the first episode. J Clin Exp Neuropsychol. 2006;28:270-82.

15. Lyoo IK, Yoon S, Kim TS, Lim SM, Choi Y, Kim JE et al. Predisposition to and effects of methamphetamine use on the adolescent brain. Mol Psychiatry. 2015 20:1516-24.

16. Mesulam, M (2000). Behavioral neuroanatomy: large-scalenetworks, association cortex, frontal syndromes, the limbic system, and hemispheric specializations. In Principles of behavioral and cognitive neurology (ed.M Mesulam), pp. 1-120. Oxford University Press: Oxford.

17. Okita, K, Morales, AM, Dean, AC, Johnson, MC, Lu, V, Farahi, J, Mandelkern, MA \& London, ED (2017). Striatal dopamine D1-type receptor availability: no difference from control but association with cortical thickness in methamphetamine users. Mol Psychiatry.

18. First MB, Spitzer RL, Gibbon M, Williams JBW. The structured clinical interview for DSM-IV axis I disorders (SCID-IP). Washington, D.C: American Psychiatric Press; 1995.

19. U.S. Department of Education, I. o. E. S. (2009). The 2009 High School Transcript Study. National Center for Education Statistics.

20. Slick D, Hopp G, Strauss E, Thompson G. The victoria symptom validity test Odessa, FL: Psychological Assessment Resources; 1997.

21. Babikian T, Boone KB, Lu P, Arnold G. Sensitivity and specificity of various digit span scores in the detection of suspect effort. Clin Neuropsychol. 2006;20:145-59.

22. Iverson GL, Lange RT, Green P, Franzen MD. Detecting exaggeration and malingering with the trail making test. Clin Neuropsychol. 2002;16:398-406.

23. Reitan RM. Validity of the trail making test as an indicator of organic brain damage. Percept Mot Skills. 1958;8:271-6.

24. Golden CJ. Stroop color and word test: a manual for clinical and experimental uses. Wood Dale, IL: Stoelting Company; 1978.

25. Wechsler, D (1997). Wechsler Adult Intelligence Scale Third Edition. The Psychological Corporation: San Antonio,TX.

26. Robertson $C L$, Ishibashi $K$, Mandelkern MA, Brown AK, Ghahremani DG, Sabb F et al. Striatal D1- and D2-type dopamine receptors are linked to motor response inhibition in human subjects. J Neurosci. 2015;35:5990-7.

27. Bilder RM, Sabb FW, Cannon TD, London ED, Jentsch JD, Parker DS et al. Phenomics: the systematic study of phenotypes on a genome-wide scale. Neuroscience. 2009;164:30-42.

28. Heaton RK. Wisconsin card sorting test manual. Odessa, FL: Psychological Assessment Resources; 1981.

29. Schretlen DJ. Brief test of attention professional manual. Odessa, FL: Psychological Assessment Resources, Inc; 1997.

30. Glahn DC, Therman S, Manninen M, Huttunen M, Kaprio J, Lonnqvist J et al. Spatial working memory as an endophenotype for schizophrenia. Biol Psychiatry. 2003;53:624-6.

31. Buschke H. Retention in immediate memory estimated without retrieval. Science. 1963;140:56-57.

32. Rey A. L'examen clinique en psychologie [Clinical tests in psychology]. Paris: Presses Universitaires de France; 1964.

33. Benedict RH. Brief visuospatial memory test-revised. Odessa, FL: Psychological Assessment Resources; 1997.

34. Delis DC, Kaplan E, Kramer JH. Delis-Kaplan executive function system (DKEFS): examiner's manual. San Antonio, TX: The Psychological Corporation; 2001.

35. Fan J, McCandliss BD, Fossella J, Flombaum J, Posner MI. The activation of attentional networks. Neuroimage. 2005;26:471-9.

36. Logan GD, Cowan WB, Davis KA. On the ability to inhibit simple and choice reaction time responses: a model and a method. J Exp Psychol Hum Percept Perform. 1984;10:276-91.

37. Dean AC, Kohno M, Morales AM, Ghahremani DG, London ED. Denial in methamphetamine users: associations with cognition and functional connectivity in brain. Drug Alcohol Depend. 2015;151:84-91.

38. Simon SL, Dean AC, Cordova X, Monterosso JR, London ED. Methamphetamine dependence and neuropsychological functioning: evaluating change during early abstinence. J Stud Alcohol Drugs. 2010;71:335-44.

39. Dean AC, Hellemann G, Sugar CA, London ED. Educational attainment is not a good proxy for cognitive function in methamphetamine dependence. Drug Alcohol Depend. 2012;123:249-54.

40. Mitrushina, M, Boone, KB, Razani, J \& D'Elia, LF. eds. (2005). Handbook of normative data for neuropsychological assessment. Oxford University Press: Oxford. 
41. McGregor C, Srisurapanont M, Mitchell A, Longo MC, Cahill S, White JM. Psychometric evaluation of the amphetamine cessation symptom assessment. J Subst Abus Treat. 2008;34:443-9.

42. Segonne F, Dale AM, Busa E, Glessner M, Salat D, Hahn HK et al. A hybrid approach to the skull stripping problem in MRI. Neuroimage. 2004;22: 1060-75.

43. Pluddemann A, Flisher AJ, McKetin R, Parry CD, Lombard CJ. A prospective study of methamphetamine use as a predictor of high school non-attendance in Cape Town, South Africa. Subst Abus Treat Prev Policy. 2010;5:25.

44. Wechsler D. Wechsler test of adult reading. San Antonio, TX: Psychological Corporation; 2001.

45. Hedges LV, Laine RD, Greenwald R. Does money matter? A meta-analysis of studies of the effects of differential school inputs on student outcomes. Educ Res. 1994;23:5-14.

46. Sayegh P, Arentoft A, Thaler NS, Dean AC, Thames AD. Quality of education predicts performance on the wide range achievement test-4th edition word reading subtest. Arch Clin Neuropsychol. 2014;29:731-6.

47. Jernigan TL, Gamst AC, Archibald SL, Fennema-Notestine C, Mindt MR, Marcotte TD et al. Effects of methamphetamine dependence and HIV infection on cerebral morphology. Am J Psychiatry. 2005;162:1461-72.
48. Kim SJ, Lyoo IK, Hwang J, Chung A, Hoon SY, Kim J et al. Prefrontal grey-matter changes in short-term and long-term abstinent methamphetamine abusers. Int J Neuropsychopharmacol. 2006;9:221-8.

49. Morales AM, Lee B, Hellemann G, O'Neill J, London ED. Gray-matter volume in methamphetamine dependence: cigarette smoking and changes with abstinence from methamphetamine. Drug Alcohol Depend. 2012;125:230-8.

50. Nakama H, Chang L, Fein G, Shimotsu R, Jiang CS, Ernst T. Methamphetamine users show greater than normal age-related cortical gray matter loss. Addiction. 2011;106:1474-83.

51. Schwartz DL, Mitchell AD, Lahna DL, Luber HS, Huckans MS, Mitchell SH et al. Global and local morphometric differences in recently abstinent methamphetaminedependent individuals. Neuroimage. 2010;50:1392-401.

52. Thompson PM, Hayashi K, Simon SL, Geaga JA, Hong MS, Sui $Y$ et al. Structural abnormalities in the brains of human subjects who use methamphetamine. J Neurosci. 2004;24:6028-36.

53. Dean AC, Groman SM, Morales AM, London ED. An evaluation of the evidence that methamphetamine abuse causes cognitive decline in humans. Neuropsychopharmacology. 2013;38:259-74.

54. Cohen J. Statistical power analysis for the behavioral sciences. Hillsdale, NJ: Lawrence Erlbaum Associates; 1988. 